\title{
Functionalized gold nanoparticles for the detection of nitrates in water
}

\author{
S. Mura · G. Greppi · P. P. Roggero • \\ E. Musu $\cdot$ D. Pittalis $\cdot$ A. Carletti · \\ G. Ghiglieri · J. Irudayaraj
}

Received: 20 February 2013/Revised: 6 November 2013/Accepted: 14 December 2013/Published online: 5 February 2014

(C) Islamic Azad University (IAU) 2014

\begin{abstract}
A rapid and sensitive colorimetric assay was developed using cysteamine modified gold nanoparticles for the direct detection of nitrates in water samples. Gold nanoparticles stabilized with citrate were modified with cysteamine that has excellent affinity for nitrates, and its capacity to capture nitrates was evaluated, in comparison with other anions. The presence of nitrates in water samples could be tracked by naked eye with a color variation of the colloidal suspension from red to gray, and these results were confirmed through ultraviolet-visible measurements within a nitrate concentration of $35 \mathrm{ppm}$. In field analysis was performed in underground water extracted from wells during the year 2012 in Arborea area (Italy), a nitrate vulnerable zone, and information of nitrate concentration in the range of the recommended nitrate level in water was studied. This simple assay can be used for onsite detection of nitrates in water without the need for skilled personnel, sample pretreatment or expensive instrumentation.
\end{abstract}

Electronic supplementary material The online version of this article (doi:10.1007/s13762-013-0494-7) contains supplementary material, which is available to authorized users.

S. Mura $(\bowtie) \cdot$ P. P. Roggero · D. Pittalis · A. Carletti Nucleo di Ricerca sulla Desertificazione, Università degli Studi di Sassari, Viale Italia 39, 07100 Sassari, Italy

e-mail: mura.stefania@gmail.com

G. Greppi

Dipartimento di Agraria, Lab. Bionanotecnologie, Università degli Studi di Sassari, Via Enrico De Nicola 9, 07100 Sassari, Italy

E. Musu

Laboratorio di Telemicroscopia Industriale, Sardegna Ricerche, Edificio 3, Loc. Piscinamanna, 09010 Pula, CA, Italy
Keywords Nanoparticles $\cdot$ Nitrates $\cdot$ Ultraviolet-visible $\cdot$ Colorimetric test kit $\cdot$ Water

\section{Introduction}

Nitrate $\left(\mathrm{NO}_{3}{ }^{-}\right)$and nitrite $\left(\mathrm{NO}_{2}{ }^{-}\right)$anions are found in the environment and can be produced in soil, water and plants through the oxidation of nitrogen by microorganisms (Addiscott and Benjamin 2004; Environment Canada 2003; Kirmeyer et al. 1995; NRC 1995; US EPA 2002). Their presence in the environment is due to their use as inorganic fertilizers, food preservatives, component of explosives and products of chemical industries; they are very stable and different nitrogenous compounds are converted into nitrates. In particular, nitrate salts are very soluble and mobile in soil and can migrate to the water table when present in excess to be used as plant nutrients (Adam 1980; Agriculture Canada 1991; Fanning 2000). The presence of nitrates in water supplies can therefore be used as an index of water pollution due to the excessive use of fertilizers, livestock waste and pollution from sewage on agricultural effluents. The problem of groundwater contamination by

\footnotetext{
D. Pittalis · A. Carletti

Department of Science for Nature and Environmental Resources, University of Sassari, 39, viale Italia, 07100 Sassari, Italy

G. Ghiglieri

Dipartimento di Scienze Chimiche e Geologiche, Laboratorio TeleGis, Università degli Studi di Cagliari, Via Trentino 51, 09127 Cagliari, Italy

J. Irudayaraj

Department of Agricultural and Biological Engineering and Bindley Bioscience Center, Purdue University, 225 S. University Street, West Lafayette 47907, Indiana
} 
nitrate ion is experienced by several countries worldwide, and different studies suggest that the nitrate concentration in water is above the limits allowed by the European Community for drinking water $(50 \mathrm{ppm}$, maximum concentration admissible CMA) and exceeds the maximum contaminant level MCL ( $45 \mathrm{ppm} \mathrm{NO} \mathrm{N}_{3}$ ) determined by the Environmental Protection Agency (EPA) (US EPA 1995; US EPA 1999; Zuane 1996). Nitrate contamination of groundwater is a problem because of its effect on birth defects, abortions, methaemoglobinaemia known as blue baby syndrome (Beatson 1978) and problems of the central nervous system (Brender and Olive 2004) in humans (Bruningfann and Kaneene 1993) and animals. Different methods exist to test the nitrate concentration based on an indirect determination after reduction to nitrites or using different techniques comprising of spectrophotometry, spectrofluorimetry, colorimetry (Griess reaction), gas chromatography-mass spectrometry, Raman spectrometry, high-performance liquid chromatography, ion chromatography, capillary electrophoresis, chemiluminescence and electrochemistry (Shaviv et al. 2003; Wang et al. 2009; Xiao et al. 2011). All of these methods are selective and sensitive but require sample pretreatment, and use of toxic reagents, furthermore they suffer from interferences, is time consuming and require sophisticated expensive instruments and highly trained technicians for the measurements. The classical methods of analysis of nitrates can not be applied in an on-site setting, in rural and remote areas where these tests are necessary. To overcome these limitations, a procedure to monitor nitrates in situ, without sample preparation, in real time, not affected by interference from competing molecules and being able to detect ppm concentrations, will be useful and practical. The earlier findings about nitrate detection provided the analysis in well-equipped laboratories, and only two recent works applied a colorimetric method for the detection of nitrites infield (Daniel et al. 2009; Xiao and Yu 2010). In these works, a direct method was used for the detection of nitrites while an indirect and complicated method was applied for the detection of nitrates. In fact in most colorimetric assays, nitrate is reduced to nitrite with enzymes or catalysts and detected indirectly with a long reaction time. The aim of the present work therefore is to develop a simple, sensitive, direct, low-cost colorimetric test kit for routine analysis to evaluate water quality and to determine ultra trace amount of nitrates using nanotechnology. Recently, gold nanoparticles have attracted great attention because of their biological compatibility and non-toxic nature (Poole and Owens 2003; Sharma et al. 2009). This interest has led to the development of biological and chemical sensors that utilize nanoparticles in different fields (catalysis, biotechnology, electronic, photonic and medicine) (Mosier-Boss and Lieberman 2002; Stewart and
Anderton 2008; Tan et al. 2010; Vongsvivut et al. 2010). Particle size and shape in fact influence physical and chemical properties of nanomaterial and provide a unique optical behavior that, according to the state of aggregation of nanoparticles, produces changes in color (Michota et al. 2002; Sharma et al. 2010; Yu et al. 2007). In fact, the greater $\lambda_{\max }$ the larger NPs dimension; larger is the peak and wider is the NPs distribution (Doering and Nie 2002; Handley 1989; Keating et al. 1999). In this effort, in this study, gold nanoparticles (AuNPs) were functionalized with a thiol (cysteamine) and their properties were studied in the presence of nitrates. The mercapto group of cysteamine could be easily attached to the surface of the AuNPs by the formation of $\mathrm{Au}-\mathrm{S}$ bonds while the $\mathrm{NH}_{2}$ groups exposed to the outer surface of NPs can interact with nitrates present in water samples. In this way, functionalized gold nanoparticles in the presence of nitrates form crosslinks that induce their aggregation and precipitation, causing a distinct change in color, visible with the naked eye. This property was exploited to develop a new disposable fast kit, composed of functionalized nanoparticles for the analysis of water samples. Taking advantage to the optical properties of functionalized nanoparticles, nitrates were also detected selectively and in a fast and simple way with ultraviolet-visible (UV-vis) measurements of water solutions. Information of nitrate concentration in the range of the recommended nitrates level in water was studied in underground water extracted from wells during the year 2012 in Arborea area (Italy), a nitrate vulnerable zone.

\section{Materials and methods}

\section{Chemicals}

Gold (III) chloride hydrate (puriss. p.a., ACS reagent, $>49 \%$ Au basis), sodium citrate dihydrate ( $>99 \%)$, calcium phosphate (purum, p.a. $>96 \%$ ), sodium carbonate (bioXtra, $>99 \%$ ), sodium bicarbonate (reagentplus $>99.5 \%$ ), sodium chloride (bioXtra, $>99.5 \%$ ), potassium nitrate (cell culture tested), calcium nitrate tetrahydrate $(>99 \%)$, cysteamine hydrochloride $(>98 \%)$ and acetone (reagent grade $>99.8 \%$ ) were purchased from Sigma-Aldrich. Deionized water (18 M $\Omega$ ) was used for the preparation of buffer solutions. All solvents and reagents were used without further purification.

Synthesis of citrate-stabilized gold nanoparticles (AuNPs)

To obtain monodispersed nanoparticles, citrate-stabilized AuNPs were synthesized with a simple equipment and with a modified method based on a Lee and Meisel's (1982) standard procedure. A solution of $\mathrm{HAuCl}_{4}(1 \mathrm{mM})$ in water 
was prepared, stirred and heated to boiling, under reflux. Then, $5 \mathrm{~mL}$ of a sodium citrate solution $(38.8 \mathrm{mM})$ was rapidly added to the solution of $\mathrm{HAuCl}_{4}$. The color of the solution changed from yellow to black, lavender and finally red. Part of this solution was continually boiled for $30 \mathrm{~min}$ and another for $3 \mathrm{~h}$. Finally, these solutions were filtered through a $0.22 \mathrm{~mm}$ Millipore syringe filter to remove the precipitate and then characterized with UV-vis and transmission electron microscopy (TEM) measurements. The filtrate was stored in refrigerator at $4{ }^{\circ} \mathrm{C}$. The synthesis of AuNPs was based on the reduction of $\mathrm{HAuCl}_{4}$ by sodium citrate. This method was used for the first time by Turkevitch in 1951 because sodium citrate acts as a reducing agent and stabilizes the anion. In fact after their formation, AuNPs are surrounded by a layer of citrate, sodium and chloride ions. Modifying citrate concentrations, nanoparticles (NPs) with a diameter between 15 and $150 \mathrm{~nm}$ can be produced, but to obtain reproducible NPs with the same size, it was important to control all the experimental conditions and the cleanliness of glassware. In the present work, the reaction was conducted at high temperature $\left(100{ }^{\circ} \mathrm{C}\right)$, with intense stirring, under reflux, and during the different steps, the change in the color of the solution was noted until the appearance of a red wine color. These color changes are due to the size and shape of NPs but also due to the refractive index of the surrounding medium and can be explained by considering the adsorption and scattering of light in the visible, both related to the resonance phenomenon of surface plasmons. Among other metals, the choice of gold is due to the fact that gold is a noble metal, inert, has a low tendency to oxidize with fewer defects compared with other metals, excellent for the formation of self-assembled monolayers (SAMs), binds the thiols with strong interactions and is biocompatible (Swaminathan et al. 2005). A functionalization step was introduced to prevent the aggregation of nanoparticles in solution. Alkyl thiols on gold have a high affinity to the surface of the metal and allow the formation of well-defined organic surfaces with variable chemical functionalities that determine the properties of the interface exposed to the external environment.

Functionalization of AuNPs with cysteamine hydrochloride $(\mathrm{Cy} \mathrm{HCl})$

For the final detection of nitrates, the citrate-stabilized AuNPs were functionalized with cysteamine, a chemical linker revealed selective for nitrate capture. This functionalization was obtained by mixing $0.001 \mathrm{M}$ cysteamine $\mathrm{HCl}$ solution in water with the AuNPs solution at a volume ratio of 1:100 and stirring for $12 \mathrm{~h}$. The final solution (AuCyNPs) appeared as a deep red color, and it was stable for several months at $4{ }^{\circ} \mathrm{C}$. UV-vis measurements were carried out in the range $400-700 \mathrm{~nm}$ to determine the maximum wavelength $\left(\lambda_{\max }\right)$ of adsorption of AuNPs and AuCyNPs in order to correlate the results with the dimensions and distribution of the NPs obtained.

\section{Detection of nitrates}

AuNPs functionalized with cysteamine (AuCyNPs) were used to link and precipitate nitrates mixing these NPs to different nitrate solutions at various concentrations and to underground water samples.

In particular, to assess the relationship between agricultural practices and quality of groundwater affected by farm nitrate contamination, different groundwater samples were taken in the specific context of the nitrate vulnerable zone (NVZ) of Arborea (Italy). In this area, characterized by sandy soils and shallow water table, zootechnical (milk production with more than 50,000 cows) and agricultural (corn and ryegrass) activities affect a fragile territory (Cau and Paniconi 2007). Since 2 years (Idrisk Project), within NVZ, a specific groundwater monitoring activity is carried out, including the wells A, B and C (Fig. 7). They have been selected for their particular pollution source properties. In fact, hydrogeological surveys, chemical and isotopical analysis suggests a direct relation between farm waste and groundwater pollution. Conventional techniques and new colorimetric techniques based on the use of functionalized gold nanoparticles were carried out. The sampling was carried out in three different wells indicated as A, B and C, from which groundwater samples were collected as illustrated in Fig. 7. The depth of the wells was A $14 \mathrm{~m}$; B $27 \mathrm{~m}$; and C $20 \mathrm{~m}$, and the distance between sites was A-B 1,400 m; B-C $730 \mathrm{~m}$; A-C 1,900 m. Two samples from each site were collected at the well-pump outflow and filtered $(0.45 \mu \mathrm{m}$ filter $)$ into $1 \mathrm{~L}$ polythene bottles thoroughly prewashed with distilled water. The samples were transported in low-temperature thermal bags and stored under refrigeration. Standard methods (APHA 1992) were applied to quantify the presence of major ions. In particular, cations $\left(\mathrm{Na}^{+}, \mathrm{K}^{+}, \mathrm{Ca}^{2+}, \mathrm{Mg}^{2+}\right)$ were determined by atomic absorption spectrometry (Perkin Elmer model AAnalist 200) while the anions $\left(\mathrm{F}^{-}, \mathrm{Cl}^{-}, \mathrm{Br}^{-}, \mathrm{NO}^{3-}, \mathrm{PO}_{4}{ }^{3-}, \mathrm{SO}_{4}{ }^{2-}\right)$ were determined by ion chromatography (anion column 20 Alltech model allsep anion $7 \mu \mathrm{m}, 100 \mathrm{~mm}$ ). Carbonate and bicarbonate contents were obtained by titration methods, whereas $\mathrm{NH}_{4}{ }^{+}, \mathrm{NO}_{2}{ }^{-}$and $\mathrm{SiO}_{2}$ content by colorimetric methods. The ion balance errors for the analyses were within $\pm 5 \%$. While for the innovative analysis with nanopartices, $0.5 \mathrm{~mL}$ of AuCyNPs $(3 \mathrm{~h})$ were mixed with $0.5 \mathrm{~mL}$ of water samples containing nitrates at different concentrations $(8-8,000 \mathrm{ppm})$, obtained dissolving $\mathrm{KNO}_{3}$ in deionized water. Different ratios of AuNPs, AuCyNPs and nitrates were tried, and also the use of aggregating agents as $\mathrm{NaCl} 0.05 \mathrm{M}$ was tested to decrease the time of reaction. In fact, in some cases, it is possible to initiate aggregation of the colloids only with the analyte, 
while, in other cases, the analyte alone is not sufficient to initiate aggregation and anionic aggregating agents are necessary (Lee and Irudayaraj 2009). To study the possible presence of interfering solutes, AuCyNPs were added to salt solutions of other anions that are usually present in water samples (phosphates, chlorides, carbonates and bicarbonates) and UV-vis spectra were analyzed. Colorimetric, UV-vis and TEM images of samples after the capture of nitrates at different concentrations were collected, and the limit of detection of the present method was evaluated on solution of nitrates and on samples of underground water collected in Arborea area. For the detection of nitrates, $0.5 \mathrm{~mL}$ of AuCyNPs ( $3 \mathrm{~h}$ ) were mixed with $0.5 \mathrm{~mL}$ of underground water and the colorimetric response was analyzed.

\section{Material characterization}

UV-visible adsorption spectra were obtained using a Nicolet Evolution 300 spectrophotometer. The spectra of nitrates and other anions were acquired from 200 to $800 \mathrm{~nm}$; a water sample was used for the background. Size and shape of AuNPs were characterized with TEM FEI Tecnai 12, and the voltage used was $120 \mathrm{kV}$. For each sample (AuNPs, AuCyNPs, AuCyNPs with nitrates), one drop of the dispersion was deposited on a copper grid. Before proceeding to the observation, the liquid was completely evaporated in $16 \mathrm{~h}$.

\section{Results and discussion}

Preparation and functionalization of AuNPs

The red gold NPs were studied with UV-visible spectroscopy and their morphology by direct visualization with TEM, so that their size and shape can be assessed. In

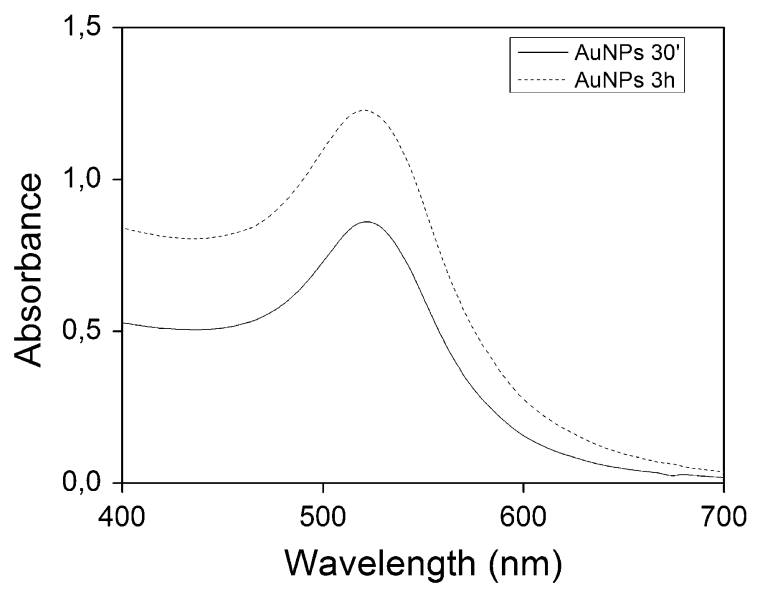

Fig. $1 \mathrm{UV}$-vis spectra of AuNPs after $30 \mathrm{~min}$ (solid line) and $3 \mathrm{~h}$ (dot line) of boiling
Fig. 1, the characteristic gold peak at $520 \mathrm{~nm}$ that indicates the formation of stable AuNPs which corresponds to radii in the range $10-30 \mathrm{~nm}$, with a narrow peak that confirms the homogeneity of size distribution can be observed. The absorbance values after $30 \mathrm{~min}$ or $3 \mathrm{~h}$ of boiling $\left(100{ }^{\circ} \mathrm{C}\right)$ indicate the different concentration of colloids prepared with different time of reaction but with the same dimension. The observed full width at half height of these gold colloidal suspensions was within $50-60 \mathrm{~nm}$. The size values and morphology of NPs were confirmed through TEM images as illustrated in Fig. 2a. The image shows monodispersed and spherical particles with an average diameter of around 10-15 $\mathrm{nm}$ in size. Gold NPs, once stabilized with sodium citrate, were modified with cysteamine (thiolated molecule) at a critical concentration, to decrease the repulsion between AuNPs to form aggregates upon sensing the presence of nitrates. As illustrated in Fig. 2b, after functionalization with cysteamine, the NPs are closer but exhibit good dispersion, while after the adsorption of high concentrations of nitrates, a strong aggregation can be noted (Fig. 2c) that can lead to NPs precipitation. In fact, after a few minutes of interaction between AuCyNPs and nitrates, the color changes can be observed either by the naked eye or from the UV spectra.

First trials for the detection of nitrates

Different UV-vis assays were performed to compare the sensitivity of the cysteamine-modified AuNPs with the direct spectra of nitrates. As reported in SI Fig. 1a (inset), UV spectra of calcium nitrate and potassium nitrate were measured at very high concentration and a peak at $300 \mathrm{~nm}$ can be observed. Decreasing the concentration of nitrates, this characteristic peak disappears, and with this method, the direct detection of nitrates at low concentration is not possible. The same test was repeated with AuCyNPs 30' (nanoparticles of gold and cysteamine boiled $30 \mathrm{~min}$ ), and also in this case, the peak at $300 \mathrm{~nm}$ was visible only at a concentration of nitrate of $8,000 \mathrm{ppm}$ (SI Fig. 1b), but observing the peak due to AuNPs at $520 \mathrm{~nm}$ at this concentration disappears as a result of formation of cluster aggregates (SI Fig. 1c), leading to a change in the color of the solution from red to gray. This property of the solution exhibiting a change in color was studied in the next sections, evaluating also the possible use of aggregating agents to facilitate the detection of nitrates at low concentration.

Selectivity of the assay toward other anions

To test the selectivity of the assay, other relevant anions (phosphates, chlorides, carbonates, bicarbonates) possibly present in water samples at high concentration were evaluated. In SI Fig. 2a is reported the UV spectrum of $\mathrm{NaCl}$ 

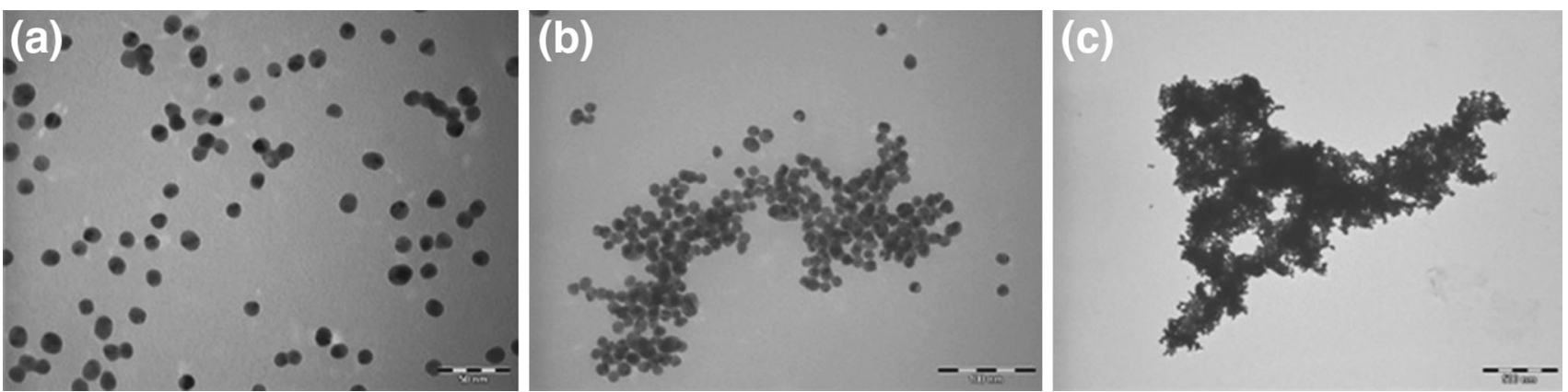

Fig. 2 TEM images of a AuNPs, b AuCyNPs, $\mathbf{c}$ AuCyNPs after the capture of nitrates at different magnification (the bar indicates $50 \mathrm{~nm}$ in fig. $2 \mathrm{a} ; 100 \mathrm{~nm}$ in fig. $2 \mathrm{~b}$ and $500 \mathrm{~nm}$ in fig. 2c)

alone without significant peaks. Adding this anion also to AuCyNPs, no changes were observed in the spectra (SI Fig. 2b) for low wavelength, while at high concentrations of chlorides $(8,000 \mathrm{ppm})$ (SI Fig. 2c), the disappearance of the peak at $520 \mathrm{~nm}$ could be noted but without a color change of the solution. In SI Fig. 3a, the phosphate spectra were reported before and after the addition to AuCyNPs solution (SI Fig. 3b), and in this case at high concentration, a peak at $270 \mathrm{~nm}$ was observed that does not have the same wavelength of the peak of nitrates, therefore not interfering. Also in this case, when this anion is added at high concentrations $(8,000 \mathrm{ppm})$ to AuCyNPs solution, the peak at $520 \mathrm{~nm}$ disappeared (SI Fig. 3c) but without any notable change in the color of the solution or other modifications. Other experiments were carried out with carbonates and bicarbonates, but the spectra were not reported because they were not significant. The developed system demonstrated a high capacity to detect nitrate ions with definite UV-vis peaks and color changes that cannot interfere with other anions present in water.

\section{Aggregation properties of AuNPs}

As illustrated in the previous sections, a significant peak and a change in color of solutions were obtained only with AuCyNPs in the presence of high concentrations of nitrates. However, the purpose of our work was to detect low concentrations of nitrates with this method. To initiate the aggregation process of nanoparticles at low concentrations of nitrates, different concentrations of aggregating agents were added to the NPs solutions. In fact, AuNPs in the colloidal suspension are negatively charged, repel each other and stay in solution. Salts such as $\mathrm{NaCl}$ shield the negative charges allowing the particles to crowd. For this reason to start the aggregation of NPs and to obtain a quick response also toward small amount of nitrates in the next experiment, AuCyNPs were added with a small amount of $\mathrm{NaCl}$ that creates a local modification of the ionic strength and enhances the interaction of AuCyNPs with $\mathrm{KNO}_{3}$ even at low concentration.

\section{Detection of nitrates with AuCyNPs}

Different trials with functionalized nanoparticles were carried out using different cysteamine molar ratios. In fact, the modification with high concentrations of cysteamine decreases the specific surface plasmon resonance of the AuNPs at $520 \mathrm{~nm}$, which leads to a minor stability of the AuNPs. Based on these results, modified AuNPs at a volume ratio cysteamine $(0.001 \mathrm{M}) / \mathrm{AuNP} 1: 100$ were used in the following experiments. In the first experiment, $0.5 \mathrm{~mL}$ of AuCyNPs (30 min) were mixed with $250 \mu \mathrm{L}$ of $\mathrm{KNO}_{3}$ solutions at different concentration in water $(8-8,000 \mathrm{ppm})$ and $125 \mu \mathrm{L}$ of $\mathrm{NaCl}(0.05 \mathrm{M})$; in this case, addition of the aggregating agent was necessary because without $\mathrm{NaCl}$ only high nitrate concentrations could be sensed with a long reaction time. As illustrated in Fig. 3, AuCyNPs

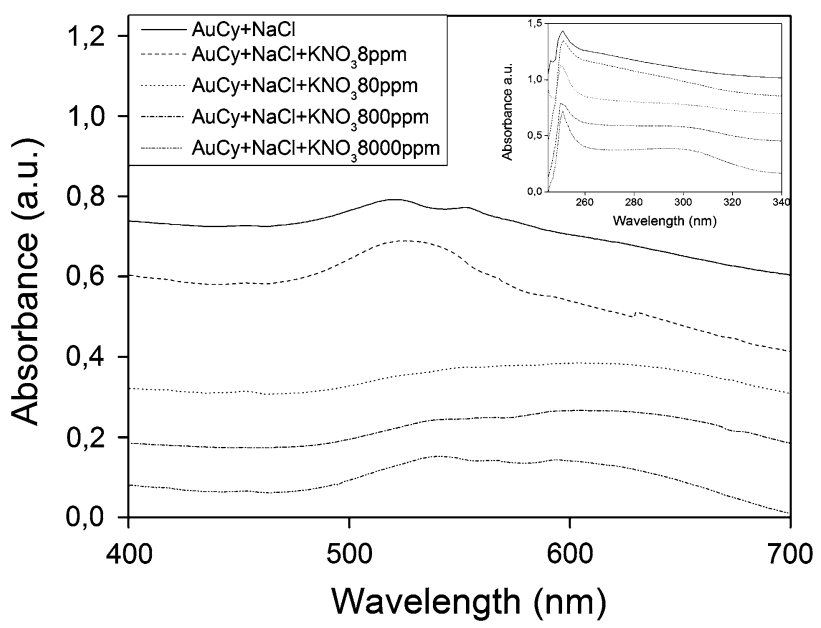

Fig. 3 UV-vis spectra of AuCyNPs boiled 30 min after the addition of chlorides and nitrates at different concentrations $(0-8,000 \mathrm{ppm}$ from the top to the bottom) in the range 400-700 $\mathrm{nm}$. Inset depicts the same measurements in the range $250-340 \mathrm{~nm}$ 
solutions present a peak at $520 \mathrm{~nm}$ due to AuNPs and a shoulder at $555 \mathrm{~nm}$ due to coupled plasmon resonance, indicating the attachment of cysteamine onto the AuNP. Increasing the concentration of nitrates, a shift of the peak at highest wavelength could be observed. From a concentration of nitrates of $80 \mathrm{ppm}$, the disappearance of the peak at $520 \mathrm{~nm}$ can be noted. At the same time, as illustrated in the inset in Fig. 3, the appearance of a peak at $300 \mathrm{~nm}$ at a concentration of $80 \mathrm{ppm}$ characteristic of nitrates can be noted. Considering the final volume of the solution with this experiment, it was possible to reach a detection limit of $35 \mathrm{ppm}$ for nitrates that is near the limits for drinking water stated by the American and European Community. This was possible using AuCyNPs with a small addition of $\mathrm{NaCl}$ as aggregating agent. This result was also confirmed colorimetrically (Fig. 5a), considering the change in the color of solutions from the reference (red AuCyNPs) with the

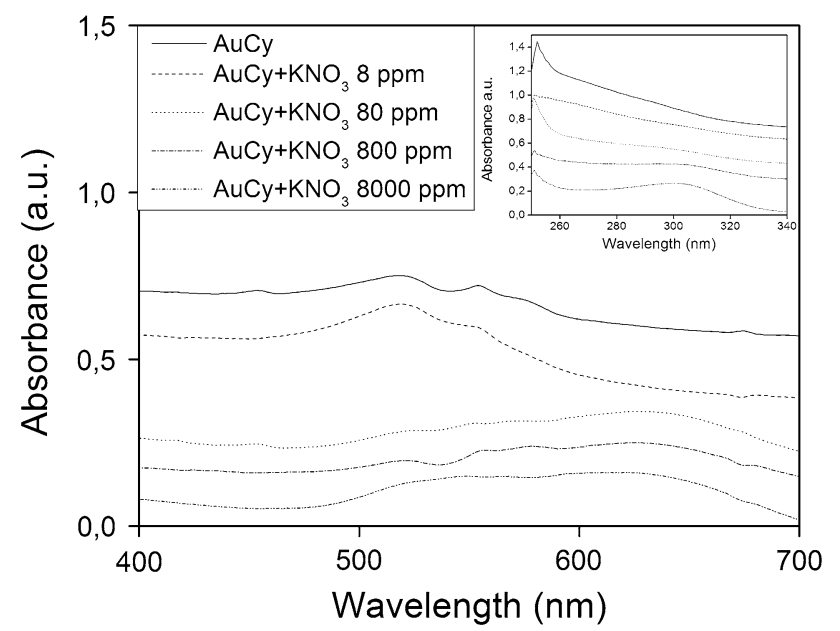

Fig. 4 UV-vis spectra of AuCyNPs boiled $3 \mathrm{~h}$ after the addition of nitrates at different concentrations $(0-8,000 \mathrm{ppm}$ from the top to the bottom) in the range $400-700 \mathrm{~nm}$. In the inset, the same measurements are depicted in the range $250-340 \mathrm{~nm}$
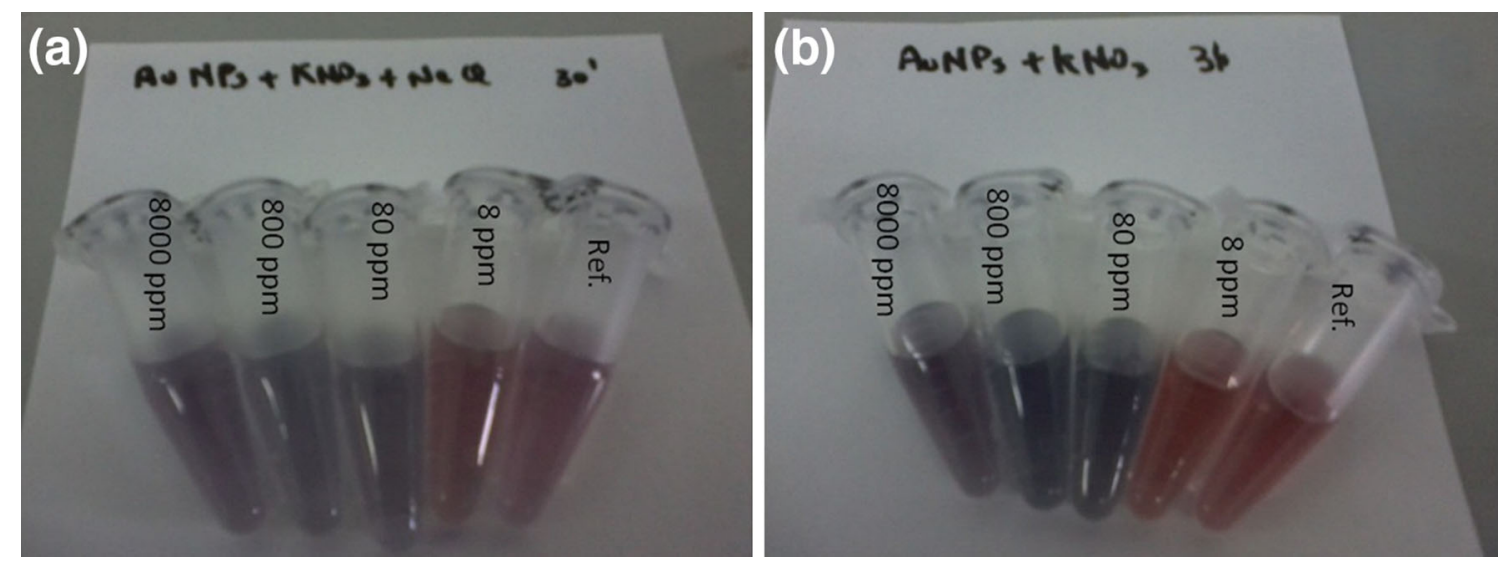

Fig. 5 Pictures of AuCyNPs alone (Ref.) and a with $\mathrm{NaCl}$ and different additions of nitrates solutions (NPs boiled 30 min) b with nitrates at different concentrations (NPs boiled $3 \mathrm{~h}$ ) 


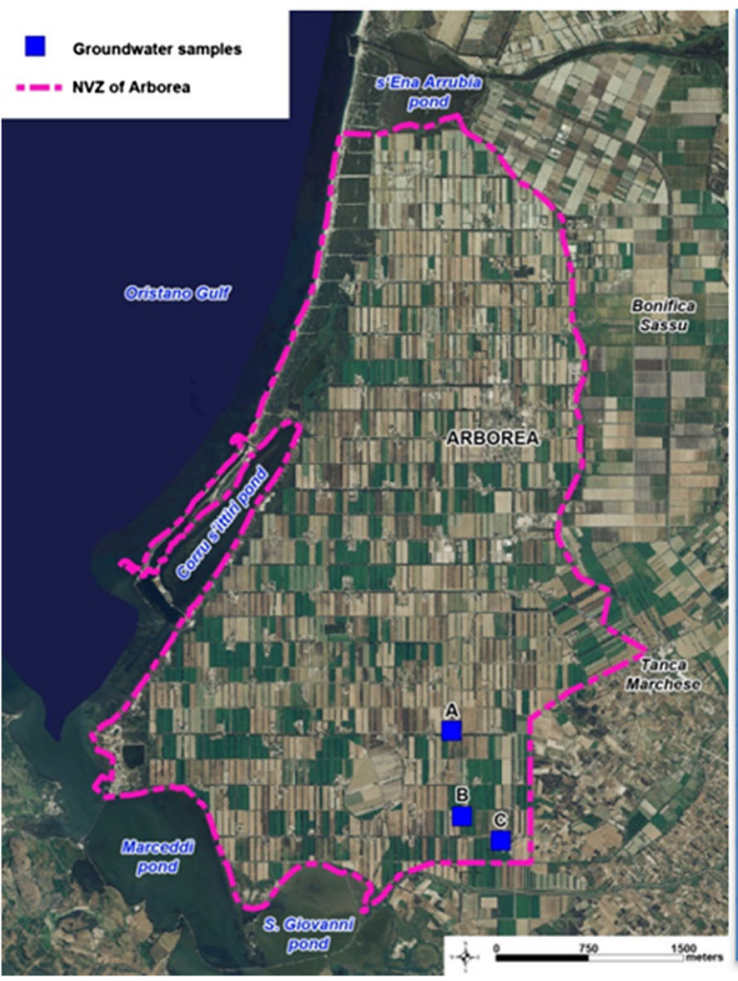

\begin{tabular}{|l|c|c|c|}
\hline & A & B & C \\
\hline $\mathrm{Ca}^{2+}$ & 27 & 82 & 218 \\
\hline $\mathrm{Mg}^{2+}$ & 17 & 60 & 125 \\
\hline $\mathrm{Na}^{+}$ & 185 & 360 & 875 \\
\hline $\mathrm{K}^{+}$ & 7.6 & 18 & 23.75 \\
\hline $\mathrm{F}^{-}$ & 0 & 0 & 0 \\
\hline $\mathrm{Cl}^{-}$ & 195.48 & 611.02 & 1809.92 \\
\hline $\mathrm{Br}^{-}$ & 0 & 0 & 0 \\
\hline $\mathrm{NO}_{3}{ }^{-}$ & 11.25 & 72.35 & 110.32 \\
\hline $\mathrm{PO}_{4}{ }^{3-}$ & 0 & 0 & 0 \\
\hline $\mathrm{SO}_{4}{ }^{2-}$ & 60.57 & 123.25 & 343.5 \\
\hline $\mathrm{HCO}_{3}^{-}$ & 296.85 & 275.13 & 222.59 \\
\hline $\mathrm{CO}_{3}{ }^{2-}$ & 0 & 0 & 0 \\
\hline $\mathrm{NH}_{4}{ }^{+}$ & 0 & 0 & 0.07 \\
\hline $\mathrm{NO}_{2}{ }^{-}$ & 0 & 0 & 0.06 \\
\hline $\mathrm{SiO}_{2}$ & 39.23 & 77.99 & 55.29 \\
\hline
\end{tabular}

Fig. 7 Map of Arborea NVZ with the wells analyzed on the left and table of cations and anions concentrations of three water samples $(A, B$ and C) expressed in $\mathrm{mg} / \mathrm{L}$ analyzed with standard methods, on the right

nitrates in solution (up to $80 \mathrm{ppm}$ ), the disappearance of the peak at $520 \mathrm{~nm}$ with the simultaneous appearance of the nitrate peak at $300 \mathrm{~nm}$ was observed (inset, Fig. 4). This particle aggregation can be observed at the same time with TEM (Fig. 2c) and colorimetric analysis (Fig. 5b), with more intense color variations compared with the previous experiment. To validate our results, the same experiment with AuCyNPs ( $3 \mathrm{~h}$ ) was carried out on three underground water samples collected from wells in a nitrate vulnerable zone. As illustrated by standard analysis (Fig. 7), these samples contain different anions and cations and therefore are useful to study the behavior of functionalized nanoparticles in presence of nitrates and other competing ions simultaneously present in solutions. The same conditions used previously with single-ion solutions were applied. As reported in Fig. 6, also in this experiment with underground water samples containing different ions, increasing the concentration of nitrates from sample A to sample C, a shift at highest wavelength in the UV spectra can be observed from a concentration of nitrates of 72-110 ppm, with a progressive disappearance of the peak at $520 \mathrm{~nm}$. Considering the final volume of suspensions also in this case, the limit of detection is $36 \mathrm{ppm}$, in accordance with the previous results. Furthermore, a color change can be observed from water samples with a nitrate concentration of $11 \mathrm{ppm}$ (inset, Fig. 6, sample A, red) to 72 and $110 \mathrm{ppm}$ (sample B and C, gray), able to discriminate water samples with a nitrate concentration over the limit for drinking water.

\section{Conclusion}

The present work consists in synthesizing gold nanoparticles functionalized with cysteamine for a direct detection of nitrates with a simple and fast method $(2 \mathrm{~min}$ for the analysis) not requiring other collateral or enzymatic reactions. AuCyNPs were synthesized and used for UV-vis and colorimetric detection of nitrates. The appeal in this colorimetric-based nanoparticle assay is its simplicity for onsite measurements. The approach does not require any instrumentation because the presence of nitrates up to $35 \mathrm{ppm}$ can be observed with a simple color change of the solution and detected by naked eye. In fact, colorimetric assay is desirable for infield monitoring due to its simplicity and portability, and with our colorimetric test kit, these results were obtained. The assay can be used to detect nitrates directly in a field setting without the need for specialized training and sample pretreatment. This proposed approach is simple, robust, sensitive, low cost, specific, selective and can be utilized for routine testing of water in the field conditions. 
Acknowledgments This work was supported by a grant financed by RAS (Regione Autonoma della Sardegna, POR Sardegna FSE 2007-2013 L.R. 7/2007, IDRISK Project, Master and Back programme). Thanks to Porto Conte Ricerche, Sardegna ricerche, University of Sassari and Purdue University for the instruments and the help provided.

\section{References}

Adam JWH (1980) Health aspects of nitrate in drinking-water and possible means of denitrification (literature review). Water SA 6:79-85

Addiscott TM, Benjamin N (2004) Nitrate and human health. Soil Use Manage 20:98-104

Agriculture Canada (1991) Canadian fertilizer consumption, shipments and trade. Policy branch, Ottawa

APHA (1992) Standard methods for examination of water and wastewater. America public health association, Washington D.C, p 19

Beatson CG (1978) Methaemoglobinaemia. Nitrates in drinking water. Environ Health 86:31-34

Brender JD, Olive JM (2004) Dietary nitrites and nitrates, nitrosatable drugs, and neural tube defects. Epidemiology 15:330-336

Bruningfann CS, Kaneene JB (1993) The effects of nitrate, nitrite and n-nitroso compounds on human health. A review. Vet Hum Toxicol 35:521-538

Cau P, Paniconi C (2007) Assessment of alternative land management practices using hydrological simulation and a decision support tool: arborea agricultural region, Sardinia. Hydrol Earth Syst Sci 11:1811-1823

Daniel WL, Han MS, Lee JS, Mirkin CA (2009) Colorimetric nitrite and nitrate detection with gold nanoparticle probes and kinetic end points. J Am Chem Soc 131:6362-6363

Doering WE, Nie SM (2002) Single-molecule and single-nanoparticle SERS: examining the roles of surface active sites and chemical enhancement. J Phys Chem B. 106:311-317

Environment Canada (2003) Canadian water quality guidelines for the protection of aquatic life: nitrate ion. National guidelines and standards office, water policy and coordination directorate, environment Canada. 115 pp. (Ecosystem health: science-based solutions report No. 1-6; dsp-psd.pwgsc.gc.ca/Collection/En134-6-2003E.pdf)

Fanning JC (2000) The chemical reduction of nitrate in aqueous solution. Coord Chem Rev 199:159-179

Handley DA (1989) Colloidal gold: principles, methods, and applications. Methods for synthesis of colloidal gold. Academic press. (1), Ch. 2

Keating C, Musick M, Keefe M, Natan M (1999) Kinetics and thermodynamics of Au colloid monolayer self-assembly. J Chem Educ 76:949-950

Kirmeyer GJ, Odell LH, Jacangelo J, Wilczak A, Wolfe R (1995) Nitrification occurrence and control in chloraminated water systems. American water works research foundation and american water works association, Denver

Lee K, Irudayaraj J (2009) Periodic and dynamic 3-D gold nanoparticle-DNA network structures for surface-enhanced raman spectroscopy-based quantification. J Phys Chem C 113:5980-5983

Lee PC, Meisel DJ (1982) Adsorption and surface-enhanced raman of dyes on silver and gold sols. J Phys Chem 86:3391-3395
Michota A, Kudelski A, Bukowska J (2002) Molecular structure of cysteamine monolayers on silver and gold substrates. Comparative studies by surface-enhanced Raman scattering. Surf Sci 502:214-218

Mosier-Boss PA, Lieberman SH (2002) Detection of anionic nutrients using cationic-coated silver SERS substrates. J Process Anal Chem 1:74-78

NRC (1995). Nitrate and nitrite in drinking water. Prepared by the subcommittee on nitrate and nitrite in drinking water, committee on toxicology, national research council. National academy press, Washington, DC

Poole CP, Owens FHJ (2003) Introduction to nanotechnology. Wiley, New York

Sharma V, Park K, Srinivasarao M (2009) Colloidal dispersion of gold nanorods: historical background, optical properties, seedmediated synthesis, shape separation and self-assembly. Mater Sci Eng R 65:1-38

Sharma A, Matharu Z, Sumana G, Solanki PR, Kim CG, Malhotra BD (2010) Antibody immobilized cysteamine functionalized-gold nanoparticles for aflatoxin detection. Thin Solid Films 519:1213-1218

Shaviv A, Kenny A, Shmulevitch I, Singher L, Aichlin YR, Katzir A (2003) Direct monitoring of soil and water nitrate by FTIR based FEWS or membrane systems. Environ Sci Technol 37:2807-2812

Stewart ME, Anderton CR (2008) Nanostructured plasmonic sensors. Chem Rev 108:494-521

Swaminathan A, Irudayaraj J, Ryan T (2005) Self-assembled monolayer-based surface plasmon immunosensor for detection of E. coli O157:H7. Biosens Bioelectron 21:998-1006

Tan J, Liu R, Wang W, Liu W, Tian Y, Wu M, Huang Y (2010) Controllable aggregation and reversible $\mathrm{pH}$ sensitivity of AuNPs regulated by carboxymethyl cellulose. Langmuir 26(3):2093-2098

Turkevich J, Stevenson PC, Hillier J (1951) A study of the nucleation and growth processes in the synthesis of colloidal gold. Discuss Faraday Soc 11:55-75

US EPA (1995) National primary drinking water regulations: contaminant specific fact sheets, inorganic chemicals, consumer version. Washington, DC

US EPA (1999) Drinking water and health. Washington, DC

US EPA (2002). Nitrification. Distribution system issue paper. Office of water, office of ground water and drinking water, US Environmental protection agency, Washington, DC

Vongsvivut J, Robertson EG, McNaughton D (2010) Surfaceenhanced Raman spectroscopic analysis of fonofos pesticide adsorbed on silver and gold nanoparticles. J Raman Spectrosc 41:1137-1148

Wang CC, Luconi MO, Masi AN, Fernández LP (2009) Derivatized silver nanoparticles as sensor for ultra-trace nitrate determination based on light scattering phenomenon. Talanta 77:1238-1243

Xiao N, Yu C (2010) Rapid-response and highly sensitive noncrosslinking colorimetric nitrite sensor using 4-aminothiophenol modified gold nanorods. Anal Chem 82:3659-3663

Xiao W, Xiao D, Xia J, Chen Z (2011) Fluorescent sensing of nitrite at nanomolar level using functionalized mesoporous silica. Microchim Acta 173:73-78

Yu C, Varghese L, Irudayaraj J (2007) Surface modification of CTAB capped gold nanorods to make molecular probes. Langmuir 23:9114-9119

Zuane JD (1996) Handbook of drinking water quality, second edn. Wiley, New York 